\title{
VERMA MODULES OVER VIRASORO-LIKE ALGEBRAS
}

\author{
XIAN-DONG WANG ${ }^{\varpi}$ and KAIMING ZHAO
}

(Received 5 April 2004; revised 11 January 2005)

Communicated by J. Du

\begin{abstract}
Let $\mathbb{K}$ be a field of characteristic $0, G$ the direct sum of two copies of the additive group of integers. For a total order $\prec$ on $G$, which is compatible with the addition, and for any $\dot{c}_{1}, \dot{c}_{2} \in \mathbb{K}$, we define $G$-graded highest weight modules $M\left(\dot{c}_{1}, \dot{c}_{2}, \prec\right)$ over the Virasoro-like algebra $\tilde{L}$, indexed by $G$. It is natural to call them Verma modules. In the present paper, the irreducibility of $M\left(\dot{c}_{1}, \dot{c}_{2}, \prec\right)$ is completely determined and the structure of reducible module $M\left(\dot{c}_{1}, \dot{c}_{2}, \prec\right)$ is also described.
\end{abstract}

2000 Mathematics subject classification: primary 17B10, 17B65, $17 \mathrm{~B} 68$.

Keywords and phrases: Virasoro-like algebra, Verma module.

\section{Introduction}

In [8], two classes of Lie algebras were defined, one is the Virasoro-like algebra, and the other one is the class of Lie algebras from quantum tori (also called $q$-deformations of the Virasoro-like algebra) which were studied in several references, for example, $[1,3,4,14]$. Generalizations of the Virasoro-like algebra were introduced and studied in [2], where their central extensions and derivations were determined. In the present paper, we are going to study representations of the Virasoro-like algebra.

Let us first recall the definition of the Virasoro-like algebra, and then describe the results in this paper. We shall assume in this paper that vector spaces are over a field $\mathbb{K}$ of characteristic 0 .

Let $G=\mathbb{Z} \oplus \mathbb{Z}$ be the direct sum of two copies of the additive group of integers. Any element $x$ in $G$ will be written as an integral vector $x=\left(x^{(1)}, x^{(2)}\right)$. The Virasorolike algebra $L=L(G)$ (over $\mathbb{K}$ ) is by definition the Lie algebra with the $\mathbb{K}$-basis

Research supported by the NSF of China (Grant No. 10171064).

(C) 2006 Australian Mathematical Society 1446-7887/06 $\$$ A2.00+0.00 
$\left\{L_{x} \mid x \in G-\{0\}\right\}$ and the Lie bracket defined as follows:

$$
\left[L_{x}, L_{y}\right]=\operatorname{det}\left(\begin{array}{l}
y \\
x
\end{array}\right) L_{x+y}, \quad \forall x, y \in G-\{0\},
$$

where

$$
x=\left(x^{(1)}, x^{(2)}\right), \quad y=\left(y^{(1)}, y^{(2)}\right), \quad\left(\begin{array}{l}
y \\
x
\end{array}\right)=\left(\begin{array}{ll}
y^{(1)} & y^{(2)} \\
x^{(1)} & x^{(2)}
\end{array}\right) .
$$

It is well known that $L$ is a simple Lie algebra.

From [2, Theorem 5.1], we know that the universal central extension of $L$ is $\bar{L}=\bar{L}(G)=L \oplus \mathbb{K} c_{1} \oplus \mathbb{K} c_{2}$, with the following Lie bracket:

$$
\begin{aligned}
{\left[c_{i}, L\right] } & =0, \quad i=1,2, \\
{\left[L_{x}, L_{y}\right] } & =\operatorname{det}\left(\begin{array}{l}
y \\
x
\end{array}\right) L_{x+y}+\delta_{x,-y}\left(x^{(1)} c_{1}+x^{(2)} c_{2}\right), \quad \forall x, y \in G-\{0\} .
\end{aligned}
$$

Actually, for any positive integer $n$, Virasoro-like algebras indexed by $\mathbb{Z}^{n+1}$, called Block algebras, were defined in [2].

It is clear that $\bar{L}$ has a natural $G$-gradation

$$
\bar{L}=\oplus_{x \in G} \bar{L}_{x}, \quad \bar{L}_{0}=\mathbb{K} c_{1}+\mathbb{K} c_{2}, \quad \bar{L}_{x}=\mathbb{K} L_{x} \quad \text { for } x \in G \backslash\{0\} .
$$

But this is not a triangular decomposition in the classical sense of [10]. Nevertheless, once we fix a total order ' $\prec$ ' on $G$ compatible with the group structure (as did in $[5,9]$ ), then we have a triangular decomposition (see Lemma 2.1). Consequently we are able to define $G$-graded highest weight modules $M\left(\dot{c}_{1}, \dot{c}_{2}, \prec\right)$ for any $\dot{c}_{1}, \dot{c}_{2} \in \mathbb{K}$ which are called Verma modules over $\bar{L}$ in Section 2. On the other hand, since we have a lot of compatible total orders on $G$ we have different Verma modules for same $\dot{c}_{1}, \dot{c}_{2} \in \mathbb{K}$. So in Section 2, we deduce some nice properties about dense total orders on $G$ (see Lemma 2.1), which will be used in later proofs.

In Section 3, using similar methods used in [5] for generalized Virasoro algebra case (certainly some different techniques have to be created) we prove that if the total order ' $\prec$ ' of $G$ is dense (see the definition in Section 2) and $\left(\dot{c}_{1}, \dot{c}_{2}\right) \neq(0,0)$, then Verma module $M\left(\dot{c}_{1}, \dot{c}_{2}, \prec\right)$ over the Virasoro-like algebra $\bar{L}$ is irreducible; if ' $\prec$ ' is dense and $\left(\dot{c}_{1}, \dot{c}_{2}\right)=(0,0)$, then $M\left(\dot{c}_{1}, \dot{c}_{2}, \prec\right)$ has a unique proper submodule with codimension 1; if ' $\prec$ ' is discrete (suppose $a=\left(a^{(1)}, a^{(2)}\right)$ is the minimal positive element) and $a^{(1)} \dot{c}_{1}+a^{(2)} \dot{c}_{2} \neq 0$, then $M\left(\dot{c}_{1}, \dot{c}_{2}, \prec\right)$ is irreducible; and it is reducible for other cases (see Theorem 3.1). We also show that for a non-trivial highest weight the unique simple quotient of $M\left(\dot{c}_{1}, \dot{c}_{2}, \prec\right)$ has an infinite-dimensional weight space.

We hope our results in this paper can have some applications in physics since the Lie algebra studied in the present paper has similar properties as the Virasoro algebra and Heisenberg algebras. 
Some non-highest weight modules over $L$ with 1-dimensional homogeneous subspaces were constructed in [12], while another kind of highest weight modules were introduced and studied in [13] where the $\mathbb{Z}$-gradation is with respect to the first index and the homogeneous subspaces are infinite dimensional.

\section{Verma modules and dense orders}

In this section we shall define Verma modules over $\bar{L}$ associated with a total order $\prec$ on $G$, and deduce some properties for dense orders on $G$.

We fix a total order $\prec$ on $G$ which is compatible with the addition, that is, $x \prec y$ implies $x+z \prec y+z$ for any $z \in G$. By $x \succ y$ we mean that $y \prec x$. The symbols $x \succeq y, x \preceq y$ have the obvious meanings. Let

$$
G_{+}:=\{x \in G \mid x \succ 0\}, \quad G_{-}:=\{x \in G|x \prec 0| .
$$

Then $G=G_{+} \cup\{0\} \cup G_{-}$. It is clear that, for any $x \in G_{+}$,

$$
\text { the set }\left\{y \in G_{+} \mid m y=x \text {, for some } m \in \mathbb{N}\right\} \text { is finite. }
$$

We denote the minimal element with respect to the order in this set by $\mu(x)$. Set

$$
\bar{L}_{+}=\sum_{x \in G_{+}} L_{x}, \quad \bar{L}_{-}=\sum_{x \in G_{-}} L_{x}, \quad \bar{L}_{0}=\mathbb{K} c_{1} \oplus \mathbb{K} c_{2} .
$$

Then we have the triangular decomposition of $L$

$$
\bar{L}=\bar{L}_{-} \oplus \bar{L}_{0} \oplus \bar{L}_{+} \text {. }
$$

The universal enveloping algebra $U=U(\bar{L})$ of $\bar{L}$ can be factored as

$$
U(\bar{L})=U\left(\bar{L}_{-}\right) \otimes U\left(\bar{L}_{0}\right) \otimes U\left(\bar{L}_{+}\right) .
$$

As for Kac-Moody algebras [6] or the Virasoro algebra [7], we can use the standard method to define Verma modules over $\bar{L}$ with respect to the above decomposition. For any $\dot{c}_{1}, \dot{c}_{2} \in \mathbb{K}$, let $I\left(\dot{c}_{1}, \dot{c}_{2}, \prec\right)$ be the left ideal of $U$ generated by the elements

$$
\left\{L_{x} \mid x>0\right\} \cup\left\{c_{1}-\dot{c}_{1}, c_{2}-\dot{c}_{2}\right\} .
$$

Then the Verma module over $\bar{L}$, associated with $\prec$, with parameters $\left(\dot{c}_{1}, \dot{c}_{2}\right)$, is defined as $M\left(\dot{c}_{1}, \dot{c}_{2}, \prec\right):=U / I\left(\dot{c}_{1}, \dot{c}_{2}, \prec\right) . L_{-x_{1}} L_{-x_{2}} \cdots L_{-x_{k}} v, k \in \mathbb{N} \cup\{0\}, x_{j} \in G$, and $x_{k} \succeq \cdots \succeq x_{2} \succeq x_{1} \succ 0$. Note that $M\left(\dot{c}_{1}, \dot{c}_{2}, \prec\right)$ is the highest weight module over $\bar{L}$ in the sense that $M\left(\dot{c}_{1}, \dot{c}_{2}, \prec\right)=\bigoplus_{x \preceq 0} M_{x}$ where $M_{0}=\mathbb{K} v$, and $M_{x}$ for $x \prec 0$ is 
spanned by $L_{-x_{1}} L_{-x_{2}} \cdots L_{-x_{k}} v$, with $k \in \mathbb{N}, x_{j} \in G$, and $x_{k} \succeq \cdots \succeq x_{2} \succeq x_{1} \succ 0$ and $x_{1}+x_{2}+\cdots+x_{r}=-x$. It is clear that $M\left(\dot{c}_{1}, \dot{c}_{2}, \prec\right)=\bigoplus_{x \leq 0} M_{x}$ is a $G$-graded $\bar{L}$-module with respect to the gradation (1.3) and that there exists $x \in G_{+}$such that $\operatorname{dim} M_{-x}=\infty$.

We call a nonzero vector $u \in M_{x}$ a weight vector with weight $x$. We will use the following notation and properties of the order $\succ$ on $G$. The notation follows [5].

Let $B(x)$ denote the set $\{y \in G \mid 0 \prec y \prec x\}$ for any $x \in G_{+}$. The order $\succ$ is called dense if

$$
\# B(x)=\infty, \quad \text { for any } x \in G_{+} \text {, }
$$

it is called discrete if

$$
\text { there exists some } a \in G_{+} \text {, such that } B(a)=\emptyset \text {. }
$$

If the total order $>$ is discrete, we denote the smallest positive element by $a=$ $\left(a^{(1)}, a^{(2)}\right)$. Now we establish some properties of dense orders $\succ$ on $G$.

Lemma 2.1. Suppose the order $>$ on $G$ is dense.

(a) For any $x, y \in G_{+}$, we can choose an element $x_{1}$ of $B(x)$ such that $\operatorname{det}\left(\begin{array}{l}y \\ \alpha\end{array}\right) \neq 0$ for all $\alpha \in B\left(x_{1}\right)$.

(b) The order $\succ$ on $G$ is standard in the sense that for any $x, y \in G_{+}$, there exists a positive integer $n$ such that $n x>y$.

(c) There exists a series $\alpha_{n}=\left(X_{n}, Y_{n}\right) \in G_{+}$for $n \in \mathbb{N}$ such that $\alpha_{n} \succ \alpha_{n+1}$, and that, for any $\varepsilon \in G_{+}$, there exists $N \in \mathbb{N}$ satisfying $\alpha_{n} \prec \varepsilon$ for all $n>N$.

(d) Suppose $a_{i}, b_{i} \in \mathbb{Z}^{*}=\mathbb{Z} \backslash\{0\}, 1 \leq i \leq r$. Then there exists $\varepsilon \in G_{+}$such that for any $(X, Y) \in B(\varepsilon), a_{i} X+b_{i} Y \neq 0,1 \leq i \leq r$.

REMARK. Orders satisfying the condition in (b) are also called Archimedean (see [11]).

PRoOF. (a) Without loss of generality, we may assume that $x=\mu(x)$ and $y=\mu(y)$. Let $x_{1}=\min \{\mu(x), \mu(y)\}$, then $x_{1}{ }^{(1)}, x_{1}{ }^{(2)}$ (and also $y_{1}{ }^{(1)}, y_{1}{ }^{(2)}$ ) are relatively prime and $B\left(x_{1}\right) \subseteq B(x)$. If $\alpha \in B\left(x_{1}\right)$ and $\operatorname{det}\left(\begin{array}{l}y \\ \alpha\end{array}\right)=0$, since $y=\mu(y)$ we then deduce that there exists a positive integer $k$ such that $\alpha=k y$, which contradict the fact that $\alpha \prec y$. Thus $\operatorname{det}\left(\begin{array}{l}y \\ \alpha\end{array}\right) \neq 0$ for any $\alpha \in B\left(x_{1}\right)$.

(b) Suppose there exist $x, y_{1} \in G_{+}$such that $n x \prec y_{1}$ for any $n \in \mathbb{N}$. Set

$$
T(x)=\left\{\sum_{i=1}^{m} a_{i} x_{i} \mid a_{i} \in \mathbb{Z}, 0 \prec x_{i} \preceq x, 1 \leq i \leq m, m \in \mathbb{N}\right\} .
$$


It is easy to see that $T(x)$ is a proper subgroup of $G, T(x) \bigcap \mathbb{Z} y_{1}=0$, and hence $T(x) \cong \mathbb{Z}$. From the definition of $T(x)$ we know that for any $y \in G_{+}, y \notin T(x)$, we have $x \prec y$. Thus the minimal positive element of $T(x)$ is also the minimal positive element in $G$. This contradicts the assumption of the lemma. So (b) follows.

(c) For any $x \in G_{+}$, choose $y^{\prime} \in B(x)$. If $2 y^{\prime} \preceq x$, we set $y=y^{\prime}$, otherwise we set $y=x-y^{\prime}$. It is clear that $2 y \prec x$. Thus we have proved that for any $x \in G_{+}$, there always exists $y \in G_{+}$such that $2 y \preceq x$. Thus we can choose a series $\alpha_{n}=\left(X_{n}, Y_{n}\right) \in G_{+}$for $n \in \mathbb{N}$ such that $\alpha_{n} \succeq 2 \alpha_{n+1}$ for all $n \in \mathbb{N}$. We claim that this series is what we are looking for. Indeed, for any $\varepsilon \in G_{+}$, from (b) we know that there exists $N \in \mathbb{N}$ such that $2^{N} \varepsilon \succ \alpha_{0}$, which implies $\varepsilon \succ \alpha_{N}$. Therefore (c) is proved.

(d) We may assume that $\left(b_{i}, a_{i}\right) \in G_{+}$for $i=1,2, \ldots, r$. Using (a) we can easily derive (d).

\section{Irreducibility of Verma modules}

In this section, we shall determine the necessary and sufficient conditions for the Verma module $M\left(\dot{c}_{1}, \dot{c}_{2}, \prec\right)$ to be an irreducible $\bar{L}$-module. Note that we do not require the modules to be $G$-graded irreducible. Actually, if $M\left(\dot{c}_{1}, \dot{c}_{2}, \prec\right)$ is irreducible, then it is certainly $G$-graded simple, but the converse is generally not true.

The method we are going to use is similar to the one used in [5] for generalized Virasoro algebra case. Since the Virasoro-like algebra and generalized Virasoro algebras have very different structure, we have to create some new techniques.

The main result of the paper is the following theorem.

THEOREM 3.1. Let $\dot{c}_{1}, \dot{c}_{2} \in \mathbb{K}$.

(1) Suppose that the order ' $\succ$ ' on $G$ is dense. Then $M\left(\dot{c}_{1}, \dot{c}_{2}, \prec\right)$ is an irreducible $\bar{L}$-module if and only if $\left(\dot{c}_{1}, \dot{c}_{2}\right) \neq(0,0)$. Moreover, $M^{\prime}(0,0, \prec):=\sum_{x \prec 0} M_{x}$, is an irreducible submodule of $M(0,0, \prec)$ of codimension 1.

(2) Suppose that the order ' $\succ$ ' on $G$ is discrete. Then $M\left(\dot{c}_{1}, \dot{c}_{2}, \prec\right)$ is an irreducible $\bar{L}$-module if and only if $a^{(1)} \dot{c}_{1}+a^{(2)} \dot{c}_{2} \neq 0$, where $a=\left(a^{(1)}, a^{(2)}\right)$ is the minimal positive element in $G$.

Proof. (1) Denote the highest weight vector in $M\left(\dot{c}_{1}, \dot{c}_{2}, \prec\right)$ by $v$. Let $u_{0} \neq 0$ be any given weight vector in $M\left(\dot{c}_{1}, \dot{c}_{2}, \prec\right)$, that is, $u_{0} \in M_{x_{0}}$ for some $x_{0} \in G$. For each $m \in \mathbb{N}$, set

$$
V_{m}:=\sum_{\substack{0 \leq s \leq m, y_{1}, \ldots, y_{,} \in G_{+} \\ y_{1} \leq \cdots \leq y_{s}}} \mathbb{K} L_{-y_{1}} \cdots L_{-y_{1}} v .
$$

It is clear that $L_{x} V_{m} \subseteq V_{m}$ for any $x \in G_{+}$. 
Claim 1. We have $L_{-x} v \in U(\bar{L}) u_{0}$ for any $x \in G_{+}$.

We use the sequence $\alpha_{n}=\left(X_{n}, Y_{n}\right) \in G_{+}$from Lemma 2.1 (c) such that for any $\varepsilon \in G_{+}$, there exists $N \in \mathbb{N}$ satisfying $\alpha_{n} \prec \varepsilon$ for all $n>N$. It is not difficult to see that $\lim _{n \rightarrow \infty} X_{n}=\lim _{n \rightarrow \infty} Y_{n}=\infty$. Here and later on, all limits are the ordinary ones and have nothing to do with the order on $G$. We may assume (choosing, if necessary, a subsequence) that $\lim _{n \rightarrow \infty} X_{n} / Y_{n}=\beta$ exists (maybe $\infty$ ). We divide the proof of Claim 1 into three steps as follows.

STEP 1. There exists some weight vector $u \in U(\bar{L}) u_{0}$ (of weight $\lambda$ ) such that, for some $r \in \mathbb{N}, u \equiv L_{-\varepsilon_{r}} \cdots L_{-\varepsilon_{1}} v\left(\bmod V_{r-1}\right)$, where $\varepsilon_{j} \in G_{+}, \varepsilon_{r} \preceq \cdots \preceq \varepsilon_{1}$, and $\varepsilon_{j}{ }^{(1)} / \varepsilon_{j}{ }^{(2)} \neq \beta$, for all $j$.

It is clear that $u_{0} \in V_{r} \backslash V_{r-1}$ for some $r \in \mathbb{N}$. If $r \leq 1$, our claim clearly holds. So we assume that $r>1$. Hence we have

$$
u_{0} \equiv \sum_{\substack{y_{1}, \ldots, y_{r} \in G_{+} \\ y_{1} \leq \cdots \leq y_{r}}} a_{\underline{y}} L_{-y_{1}} \cdots L_{-y_{r}} v \quad\left(\bmod V_{r-1}\right),
$$

where $\underline{y}=\left(y_{1}, \ldots, y_{r}\right)$. Let $I:=\left\{\left(y_{1}, \ldots, y_{r}\right) \mid a_{\underline{y}} \neq 0\right\}$. By our assumption, $I \neq \emptyset$ and $I$ is finite. For any $\underline{y}:=\left(y_{1}, \ldots, y_{r}\right)$, and $\underline{y}^{\prime}:=\left(y_{1}^{\prime}, \ldots, y_{r}^{\prime}\right) \in I$, we define

$$
\begin{aligned}
& \underline{y} \succ \underline{y}^{\prime} \quad \text { if and only if there exists some } 1 \leq s \leq r \text { such that } \\
& y_{s} \succ y_{s}^{\prime}, \text { and } y_{t}=y_{t}^{\prime} \text { for any } t>s .
\end{aligned}
$$

Let $\underline{x}:=\left(x_{1}, \ldots, x_{r}\right), x_{1} \preceq \cdots \preceq x_{r}$, be the unique maximal element in $l$, and

$$
\alpha_{1}=\min \left(\left\{y_{i}, y_{i}-y_{j} \mid \underline{y} \in I, i \neq j ; i, j=1,2, \ldots, r\right\} \cap G_{+}\right) .
$$

Then for any $\varepsilon_{1} \in B\left(\alpha_{1}\right), x_{r}-\varepsilon_{1} \succ y_{i}$ if $y_{i} \neq x_{r}$ for any $\underline{y}=\left(y_{1}, y_{2}, \ldots, y_{r}\right) \in I$. Then $\varepsilon_{1} \prec y_{i}$ for any $\underline{y} \in I$. By Lemma 2.1 (a) we may also assume that $\varepsilon_{1}{ }^{(1)} / \varepsilon_{1}{ }^{(2)} \neq \beta$ and $\operatorname{det}\left(\begin{array}{l}x_{r} \\ \varepsilon_{1}\end{array}\right) \neq 0$. By the formula

$$
L_{x_{r}-\varepsilon_{1}} L_{-x_{r}}^{s} v \equiv s \operatorname{det}\left(\begin{array}{c}
-x_{r} \\
-\varepsilon_{1}
\end{array}\right) L_{-\varepsilon_{1}} L_{-x_{r}}^{s-1} v \quad\left(\bmod V_{s-1}\right),
$$

and commutator relations for $L$, and noting that $x_{r}-\varepsilon_{1}-y_{1}>0$ for any $\underline{y} \in I$, it is easy to see that

$$
u_{1}:=L_{x_{r}-\varepsilon_{1}} u_{0} \equiv \sum_{\substack{y_{1}, \ldots, v_{r-1} \in G_{+} \\ y_{1} \leq \cdots \leq y_{r-1}}} a_{\underline{v}}^{(1)} L_{-\varepsilon_{1}} L_{-y_{1}} \cdots L_{-y_{r-1}} v \quad\left(\bmod V_{r-1}\right),
$$

and $u_{1} \notin V_{r-1}$. Define $I^{(1)}:=\left\{\left(\varepsilon_{1}, y_{1}, y_{2}, \ldots, y_{r-1}\right) \mid a_{y}^{(1)} \neq 0\right\} \neq \emptyset$. Moreover, $\underline{x}^{[1]}:=\left(\varepsilon_{1}, x_{1}, \ldots, x_{r-1}\right)$ is the unique maximal element in $I^{(1)}$.

In this manner we can easily prove by induction on $s$ the following statement for $s=2, \ldots, r$. 
(1) Let

$$
\alpha_{s}=\min \left(\left\{z_{i}, z_{i}-z_{j} \mid \underline{z}=\left(z_{1}, \ldots, z_{r}\right) \in I^{(s-1)}, i \neq j ; i, j=1, \ldots, r\right\} \cap G_{+}\right) .
$$

Then we can choose $\varepsilon_{s} \in B\left(\alpha_{s}\right)$ such that $x_{r-s+1}-\varepsilon_{s}>z_{i}$ if $z_{i} \neq x_{r-s+1}$ for any $\underline{z}=\left(z_{1}, z_{2}, \ldots, z_{r}\right) \in I^{(s-1)}$. We may also assume that $\varepsilon_{s}{ }^{(1)} / \varepsilon_{s}{ }^{(2)} \neq \beta$ and $\operatorname{det}\left(\begin{array}{c}x_{r-s+1} \\ \varepsilon_{s}\end{array}\right) \neq 0$.

(2) Let $u_{s}:=L_{x_{r-s+1}-\varepsilon_{s}} u_{s-1}$. Then

$$
u_{s} \equiv \sum_{\substack{y_{1} \ldots, y_{r-s} \in G_{+} \\ y_{1} \leq \cdots \leq j_{r-s}}} a_{\underline{y}}^{(s)} L_{-\varepsilon_{s}} \cdots L_{-\varepsilon_{1}} L_{-y_{1}} \cdots L_{-y_{r-s}} v\left(\bmod V_{r-1}\right),
$$

and $u_{s} \notin V_{r-1}$.

(3) Let $I^{(s)}:=\left\{\left(\varepsilon_{s}, \ldots, \varepsilon_{1}, y_{1}, \ldots, y_{r-s}\right) \mid a_{\underline{y}}^{(s)} \neq 0\right\}$. Then $I^{(s)} \neq \emptyset$. Moreover, $\underline{x}^{[s]}:=\left(\varepsilon_{s}, \ldots, \varepsilon_{1}, x_{1}, \ldots, x_{r-s}\right)$ is the unique maximal element in $I^{(s)}$.

Now our Step 1 follows immediately by letting $s=r$ with $u=u_{r}$.

STEP 2. There exists some $x \in G_{+}$such that $L_{-x} v \in U(\bar{L}) u_{0}$.

By the result of Step 1, there is a weight vector $u \in U(\tilde{L}) u_{0}$ (of weight $\lambda$ ) of the form

$$
u=L_{-\varepsilon_{r}} \cdots L_{-\varepsilon_{1}} v+\sum_{\substack{0 \leq k<r \\ y_{1} \leq \cdots \leq y_{k}}} b_{\underline{y}} L_{-y_{1}} \cdots L_{-y_{k}} v,
$$

where $y_{s}, \varepsilon_{s} \in G_{+}$, for all $s$, and $\varepsilon_{r} \prec \cdots \prec \varepsilon_{1}$. We can assume without loss of generality that $\lambda \neq 0$ (in fact, $-\lambda=\varepsilon_{1}+\varepsilon_{2}+\cdots+\varepsilon_{r}$ ).

Let $I_{0}:=\left\{\left(y_{1}, \ldots, y_{k}\right) \mid b_{\underline{y}} \neq 0\right\}, \underline{y}(0):=\min \left\{\varepsilon_{r}, y_{1} \mid \underline{y}:=\left(y_{1}, \ldots, y_{k}\right) \in I_{0}\right\}$. Let $\varepsilon \in B(y(0))$. Recall that $\bar{u}$ is a weight vector of weight $\bar{\lambda} \prec 0$. Then, using the commutation relations for $L$, we have

where

$$
\begin{aligned}
L_{-\lambda-\varepsilon} u & =f(-\lambda-\varepsilon) L_{-\varepsilon} v+\sum_{\substack{1 \leq k<r \\
y t \leq \cdots \leq y_{k}}} b_{\underline{y}} g_{\underline{y}}(-\lambda-\varepsilon) L_{-\varepsilon} v \\
& =\left\{f(-\lambda-\varepsilon)+\sum_{\substack{1 \leq k<r \\
y_{1} \leq \ldots \leq y_{k}}} b_{\underline{\underline{y}}} g_{\underline{\underline{y}}}(-\lambda-\varepsilon)\right\} L_{-\varepsilon} v,
\end{aligned}
$$

$$
\begin{aligned}
& f(-\lambda-\varepsilon) \\
& \quad=\operatorname{det}\left(\begin{array}{c}
-\varepsilon_{r} \\
-\lambda-\varepsilon
\end{array}\right) \operatorname{det}\left(\begin{array}{c}
-\varepsilon_{r-1} \\
-\lambda-\varepsilon_{r}-\varepsilon
\end{array}\right) \cdots \operatorname{det}\left(\begin{array}{c}
-\varepsilon_{1} \\
-\lambda-\varepsilon_{r}-\cdots-\varepsilon_{2}-\varepsilon
\end{array}\right), \\
& g_{\underline{y}}(-\lambda-\varepsilon) \\
& \quad=\operatorname{det}\left(\begin{array}{c}
-y_{1} \\
-\lambda-\varepsilon
\end{array}\right) \operatorname{det}\left(\begin{array}{c}
-y_{2} \\
-\lambda-y_{1}-\varepsilon
\end{array}\right) \cdots \operatorname{det}\left(\begin{array}{c}
-y_{k} \\
-\lambda-y_{1}-\cdots-y_{k-1}-\varepsilon
\end{array}\right) .
\end{aligned}
$$


Let $\varepsilon=(X, Y), \varepsilon_{i}=\left(a_{i}, b_{i}\right), 1 \leq i \leq r$, then

$$
\Delta(X, Y):=f(-\lambda-\varepsilon)+\sum_{\substack{1 \leq k<r \\ y_{1} \leq \cdots \leq y_{k}}} b_{\underline{y}} g_{\underline{y}}(-\lambda-\varepsilon)=f_{r}(X, Y)+\cdots+f_{0}(X, Y),
$$

where $f_{i}(X, Y)$ is a homogeneous polynomial of degree $i(0 \leq i \leq r)$ in terms of $X, Y$, and $f_{r}(X, Y)=\prod_{i=1}^{r}\left(a_{i} Y-b_{i} X\right)$, where $a_{i} \neq 0, b_{i} \neq 0$ are integers.

Set $Z_{n}=X_{n} / Y_{n}$ for $n \in \mathbb{N}$ (already chosen at the beginning of the proof). We see that $\left\{Z_{n} \mid n \in \mathbb{Z}\right\}$ is an infinite series consisting of rational numbers, and we have the following two cases.

Case I: $\lim _{n \rightarrow \infty} Z_{n}=\infty$.

It is clear that $\lim _{n \rightarrow \infty} Z_{n}^{-1}=0$, and

$$
\lim _{n \rightarrow \infty} \frac{f_{r}\left(X_{n}, Y_{n}\right)}{X_{n}^{r}}=(-1)^{r} \prod_{i=1}^{r} b_{i} \neq 0 .
$$

Since $\lim _{n \rightarrow \infty} f_{i}\left(X_{n}, Y_{n}\right) / X_{n}^{r}=0$ for $0 \leq i \leq r-1$ there exists $m$ such that $\Delta\left(X_{m}, Y_{m}\right) \neq 0$. Let $\varepsilon=\left(X_{m}, Y_{m}\right)$, then $0 \neq L_{-\varepsilon} v \in U(\bar{L}) u_{0}$ as required.

Case II: $\lim _{n \rightarrow x} Z_{n}=\beta \neq \infty$.

From Step 1 we know that the real number $\beta \neq a_{i} / b_{i}$ for $1 \leq i \leq r$. Thus

$$
\lim _{n \rightarrow \infty} \frac{f_{r}\left(X_{n}, Y_{n}\right)}{Y_{n}^{r}}=\prod_{i=1}^{r}\left(a_{i}-b_{i} \beta\right) \neq 0 .
$$

Since $\lim _{n \rightarrow \infty} f_{i}\left(X_{n}, Y_{n}\right) / Y_{n}^{r}=0$ for $0 \leq i \leq r-1$, there exists $m$ such that $\Delta\left(X_{m}, Y_{m}\right) \neq 0$. Let $\varepsilon=\left(X_{m}, Y_{m}\right)$, then $0 \neq L_{-\varepsilon} v \in U(\bar{L}) u_{0}$.

Thus Step 2 has been proved.

STEP 3. $L_{-x} v \in W=U(\bar{L}) u_{0}$ for all $x \in G_{+}$.

From Step 2, we know that there exists $\varepsilon \in G_{+}$, such that $L_{-\varepsilon} v \in W$. For $\varepsilon^{\prime} \in G_{+}$ with $\varepsilon^{\prime} \prec \varepsilon$ and $\operatorname{det}\left(\begin{array}{c}\varepsilon^{\prime} \\ \varepsilon\end{array}\right) \neq 0$, we have

$$
L_{-\varepsilon^{\prime}} v=\left(\operatorname{det}\left(\begin{array}{c}
\varepsilon^{\prime} \\
\varepsilon
\end{array}\right)\right)^{-1} L_{\varepsilon-\varepsilon^{\prime}} L_{-\varepsilon} v \in U(\bar{L}) u_{0} .
$$

Since

$$
\operatorname{det}\left(\begin{array}{l}
\varepsilon^{\prime} \\
\varepsilon
\end{array}\right) L_{-\left(\varepsilon^{\prime}+\varepsilon\right)} v=L_{-\varepsilon} L_{-\varepsilon^{\prime}} v-L_{-\varepsilon^{\prime}} L_{-\varepsilon} v \in U(\bar{L}) u_{0}
$$

it follows that $L_{-\left(\xi^{\prime}+\varepsilon\right)} v \in U(\bar{L}) u_{0}$. In general, using the formula

$$
\operatorname{det}\left(\begin{array}{c}
\varepsilon^{\prime} \\
\varepsilon
\end{array}\right) L_{-\left(\xi^{\prime}+(n+1) \varepsilon\right)} v=L_{-\varepsilon} L_{-\left(\varepsilon^{\prime}+n \varepsilon\right)} v-L_{-\left(\varepsilon^{\prime}+n \varepsilon\right)} L_{-\varepsilon} v,
$$


we can easily see that $L_{-\left(\varepsilon^{\prime}+n \varepsilon\right)} v \in U(\bar{L}) u_{0}$ for any $n \in \mathbb{Z}_{+}$. Since the order is standard, we deduce that for all $x \in G_{+}$, we have $n$ such that $\varepsilon^{\prime}+(n+1) \varepsilon>\varepsilon^{\prime}+n \varepsilon>x$, and at least one of the determinants $\operatorname{det}\left(\begin{array}{c}\varepsilon^{\prime}+n \varepsilon \\ x\end{array}\right)$ and $\operatorname{det}\left(\begin{array}{c}\varepsilon^{\prime}+(n+1) \varepsilon \\ x\end{array}\right)$ is not 0 , say the first one is not zero. Hence

$$
L_{-x} v=c\left(L_{-x+\varepsilon^{\prime}+n \varepsilon} L_{-\left(\varepsilon^{\prime}+n \varepsilon\right)} v\right) \in W=U(\bar{L}) u_{0},
$$

where $c$ is a nonzero constant. Step 3 is true. Thus Claim 1 follows.

Now choose $\varepsilon, \varepsilon^{\prime} \in G_{+}$, such that $\operatorname{det}\left(\begin{array}{l}\varepsilon^{\prime} \\ \varepsilon\end{array}\right) \neq 0$, we have

$$
\begin{array}{rlrl}
L_{\varepsilon} L_{-\varepsilon} v & =\left(\varepsilon^{(1)} \dot{c}_{1}+\varepsilon^{(2)} \dot{c}_{2}\right) v \in U(\bar{L}) u_{0}, & \varepsilon & =\left(\varepsilon^{(1)}, \varepsilon^{(2)}\right), \\
L_{\varepsilon^{\prime}} L_{-\varepsilon^{\prime}} v & =\left({\varepsilon^{\prime}}^{(1)} \dot{c}_{1}+\varepsilon^{\prime(2)} \dot{c}_{2}\right) v \in U(\bar{L}) u_{0}, & \varepsilon^{\prime}=\left(\varepsilon^{\prime(1)}, \varepsilon^{\prime(2)}\right)
\end{array}
$$

from which we see that the module $M\left(\dot{c}_{1}, \dot{c}_{2}, \prec\right)$ is generated by any nonzero weight vector if and only if $\left(\dot{c}_{1}, \dot{c}_{2}\right) \neq(0,0)$. On the other hand, if $\left(\dot{c}_{1}, \dot{c}_{2}\right)=(0,0)$, from Step 3, we see that

$$
M^{\prime}(0,0, \prec):=\sum_{x_{1}, \ldots, x_{k} \in G_{+}, k>0} \mathbb{K} L_{-x_{1}} \cdots L_{-x_{k}} v_{0}
$$

is generated by any nonzero weight vector with nonzero weight.

Suppose $p=v_{1}+\cdots+v_{r} \in M\left(\dot{c}_{1}, \dot{c}_{2}, \prec\right)$ where $v_{i} \in M_{x_{i}} \backslash\{0\}$ with $x_{1} \prec \cdots \prec x_{r}$. Choose $y \in G_{+}$with $x_{1} \prec-y \prec x_{2}$. We know that there exists $w \in U\left(\bar{L}_{+}\right)_{y}$ such that $w v_{1} \neq 0$. Thus $w p=w v_{1} \in U(\bar{L}) p$ which is a weight vector with weight $x_{1}+y$. Thus from Claim 1 and the above argument, part (1) follows.

(2) Suppose the order ' $\succ$ ' on $G$ is discrete. Then $a \mathbb{Z} \subseteq G$ where $a$ is as in (2.4). For any $x \in G$, we write $x \succ a \mathbb{Z}$ if $x>n a$ for any $n \in \mathbb{Z}$. Let $H_{+}:=\{x \in G \mid x>a \mathbb{Z}\}$, $H_{-}=-H_{+}$. It is not difficult to see (by (2.3)) that $G=a \mathbb{Z} \cup H_{+} \cup H_{-}$. Let $M_{a}\left(\dot{c}_{1}, \dot{c}_{2}, \prec\right)$ be the submodule of $M\left(\dot{c}_{1}, \dot{c}_{2}, \prec\right)$ generated by the highest weight vector $v$ as a module over the subalgebra $L(a \mathbb{Z})$ which is a Heisenberg algebra over $\mathbb{K}$ with the basis: $\left\{a^{(1)} c_{1}+a^{(2)} c_{2}, L_{a i} \mid i \in \mathbb{Z}\right\}$.

It is clear that $U\left(H_{+}\right) M_{a}\left(\dot{c}_{1}, \dot{c}_{2}, \prec\right)=0$. Since

$$
M\left(\dot{c}_{1}, \dot{c}_{2}, \prec\right) \cong U(\bar{L}) \bigotimes_{U\left(L(a \mathbb{Z})+H_{+}\right)} M_{a}\left(\dot{c}_{1}, \dot{c}_{2}, \prec\right),
$$

it follows that the irreducibility of $\bar{L}$-module $M\left(\dot{c}_{1}, \dot{c}_{2}, \prec\right)$ would imply the irreducibility of $L(a \mathbb{Z})$-module $M_{a}\left(\dot{c}_{1}, \dot{c}_{2}, \prec\right)$ which is a highest weight Heisenberg module with $c=a^{(1)} \dot{c}_{1}+a^{(2)} \dot{c}_{2}$. It is well known that $L(a \mathbb{Z})$-module $M_{a}\left(\dot{c}_{1}, \dot{c}_{2}, \prec\right)$ is irreducible if and only if $c \neq 0$. Thus if $M\left(\dot{c}_{1}, \dot{c}_{2}, \prec\right)$ is irreducible, then $c \neq 0$.

Conversely, suppose $a^{(1)} \dot{c}_{1}+a^{(2)} \dot{c}_{2} \neq 0$, that is, $M_{a}\left(\dot{c}_{1}, \dot{c}_{2}, \prec\right)$ is an irreducible highest weight $L(a \mathbb{Z})$-module. Let $u_{0} \notin F v$ be any nonzero weight vector in $M\left(\dot{c}_{1}, \dot{c}_{2}, \prec\right)$. Then $u_{0} \in V_{r} \backslash V_{r-1}$ for some $r \in \mathbb{N}$ where $V_{m}$ is defined as in (3.1). 
Claim 2. We have $U(\bar{L}) u_{0} \cap M_{a}\left(\dot{c}_{1}, \dot{c}_{2}, \prec\right) \neq\{0\}$.

Write

$$
u_{0} \equiv \sum_{\substack{, y_{1}^{\prime}, \ldots, y_{s}^{\prime} \in H_{+}, y_{1}, \ldots, y_{r-s} \in a \mathbb{Z}_{+} \\ y_{1}^{\prime} \leq \cdots \leq y_{s}^{\prime}, y_{1} \leq \cdots \leq y_{r-s}}} a_{\bar{y}} L_{-y_{1}^{\prime}} \cdots L_{-y_{s}^{\prime}} L_{-y_{1}} \cdots L_{-y_{r-s}} v \quad\left(\bmod V_{r-1}\right)
$$

where $\bar{y}=\left(y_{1}, \ldots, y_{r-s}, y_{1}^{\prime}, \ldots, y_{s}^{\prime}\right)$. Let $\bar{l}:=\left\{\left(y_{1}, \ldots, y_{r-s}, y_{1}^{\prime}, \ldots, y_{s}^{\prime}\right) \mid a_{\bar{y}} \neq 0\right\}$. By our construction, $\bar{I} \neq \emptyset$ and $\bar{I}$ is finite. For any $\bar{y}, \bar{z} \in \bar{I}$, we still use the definition for $\bar{y} \succ \bar{z}$ as defined in (3.2). Let

$$
\bar{x}:=\left(x_{1}, \ldots, x_{r-m}, x_{1}^{\prime}, \ldots, x_{m}^{\prime}\right), \quad x_{1}^{\prime}, \ldots, x_{m}^{\prime} \in H_{+}, x_{1}, \ldots, x_{r-m} \in a \mathbb{Z}_{+}
$$

be the unique maximal element in $\bar{I}$. Since $y_{i}^{\prime} \notin a \mathbb{Z}$, then $\operatorname{det}\left(\begin{array}{l}y_{i}^{\prime} \\ a\end{array}\right) \neq 0$, and we have

$$
\begin{aligned}
u(1) & :=L_{x_{m}^{\prime}-a} u_{0} \\
& \equiv \sum_{\substack{y_{1}^{\prime}, \ldots, y_{1-1}^{\prime} \in H_{+}, y_{1} \ldots, y_{r-1} \in a \mathbb{Z}_{+} \\
y_{1}^{\prime} \leq \cdots \leq y_{t-1}^{\prime}, y_{1} \leq \ldots \leq y_{r-1}}} a_{\bar{y}}^{(1)} L_{-y_{1}^{\prime}} \cdots L_{-y_{t-1}^{\prime}} L_{-a} L_{-y_{1}} \cdots L_{-y_{r-t}} v \quad\left(\bmod V_{r-1}\right),
\end{aligned}
$$

and $u(1) \notin V_{r-1}$. Define

$$
\bar{I}^{(1)}:=\left\{\left(a, y_{1}, \ldots, y_{r-t}, y_{1}^{\prime}, \ldots, y_{t-1}^{\prime}\right) \mid a_{\bar{y}}^{(1)} \neq 0\right\} \neq \emptyset .
$$

Let $\bar{x}^{(1)}:=\left(a, x_{1}, \ldots, x_{r-m}, x_{1}^{\prime}, \ldots, x_{m-1}^{\prime}\right)$ be the unique maximal element in $\bar{I}^{(1)}$.

Now for $s=2, \ldots, r$, we define recursively and prove by induction that

(1) Let $u(s):=L_{x_{m-s+1}^{\prime}-a} u(s-1)$. Then

$$
u(s) \equiv \sum_{\substack{y_{1}^{\prime} \ldots, y_{i-s}^{\prime} \in H_{+}, y_{1}, \ldots, y_{r-r} \in a \mathbb{Z}_{+} \\ y_{1}^{\prime} \leq \cdots \leq y_{t-s}^{+}, y_{1} \leq \cdots \leq y_{r-1}}} a_{\bar{y}}^{(s)} L_{-y_{1}^{\prime}} \cdots L_{-y_{t-s}^{\prime}} L_{-a}^{s} L_{-y_{1}} \cdots L_{-y_{r-r}} v \quad\left(\bmod V_{r-1}\right),
$$

and $u(s) \notin V_{r-1}$.

Moreover,

$$
\bar{I}^{(s)}:=\left\{(\underbrace{a, \ldots, a}_{s \text { times }}, y_{1}, \ldots, y_{r-t}, y_{1}^{\prime}, \ldots, y_{t-s}^{\prime}) \mid a_{\bar{y}}^{(s)} \neq 0\right\} \neq \emptyset
$$

$$
\bar{x}^{(s)}:=(\underbrace{a, \ldots, a}_{s \text { times }}, x_{1}, \ldots, x_{r-m}, x_{1}^{\prime}, \ldots, x_{m-s}^{\prime})
$$

is the unique maximal element in $\bar{I}^{(s)}$. 
Letting $s=m$ and noting that $u(m)$ is a weight vector, we get that $0 \neq u(m) \in$ $U(\bar{L}) u_{0} \cap M_{a}\left(\dot{c}_{1}, \dot{c}_{2}, \prec\right)$ as required. Thus Claim 2 is proved.

From Claim 2 we see that $M\left(\dot{c}_{1}, \dot{c}_{2}, \prec\right)$ is generated by any nonzero weight vector as $\bar{L}$-module.

Suppose $p=u+v_{1}+\cdots+v_{r} \in M\left(\dot{c}_{1}, \dot{c}_{2}, \prec\right)$, where $u \in U(L(\mathbb{Z} a)) v, v_{i} \in M_{x_{i}} \backslash\{0\}$ with $x_{i} \notin \mathbb{Z} a$ and $x_{1} \prec \cdots \prec x_{r}$. Choose $y \in G_{+}$with $x_{1}+y \in \mathbb{Z} a$. From Claim 2 we know that there exists $w \in U\left(\bar{L}_{+}\right)_{y}$ such that $w v_{1} \neq 0$. It is clear that $x_{i}+y \in \mathbb{Z} a$ or $x_{i}+y \succ \mathbb{Z} a$, anyway we always have $x_{1}+y \prec x_{2}+y \prec \cdots \prec x_{r}+y$. If $x_{i}+y \succ \mathbb{Z} a$ we see that $w v_{i}=0$, otherwise $w v_{i} \in U(L(\mathbb{Z} a)$ ). Thus $w p \in U(L(\mathbb{Z} a)) v$ (not necessarily a weight vector) which is a nonzero vector. Since the $L(\mathbb{Z} a)$ )-module $U(L(\mathbb{Z} a)) v$ is irreducible, Part (2) of the theorem follows.

Next we shall study reducible Verma module $M\left(\dot{c}_{1}, \dot{c}_{2}, \prec\right)$ in Theorem 3.1 (2). Without loss of generality we may assume that the order $\prec$ is defined as follows: $(i, j) \prec\left(i^{\prime}, j^{\prime}\right)$ if and only if $j<j^{\prime}$, or $j=j^{\prime}$ and $i<i^{\prime}$. We know that $a=(1,0)$ is the minimal positive element in $G$ with respect to $\prec$. From Theorem 3.1 (2) we see that $M\left(\dot{c}_{1}, \dot{c}_{2}, \prec\right)$ is reducible if and only if $\dot{c}_{1}=0$. We simply denote $M\left(0, \dot{c}_{2}, \prec\right)$ by $M\left(\dot{c}_{2}\right)$. It is clear that $M\left(\dot{c}_{2}\right)$ has a unique maximal proper $G$-graded submodule $J$. Then we have the irreducible $\bar{L}$-module $M^{\prime}\left(\dot{c}_{2}\right)=M\left(\dot{c}_{2}\right) / J$, which has the induced $G$-gradation $M^{\prime}\left(\dot{c}_{2}\right)=\bigoplus_{x \leq 0} M_{x}^{\prime}$. It is clear that $M^{\prime}(0)$ is a one-dimensional trivial $\bar{L}$-module. Next we suppose $\dot{c}_{2} \neq 0$, and easily see that

$$
L_{(k, 0)} v=0, \quad M_{(k,-1)}^{\prime}=\mathbb{K} L_{(k,-1)} v, \quad \forall k \in \mathbb{Z} .
$$

The following result tells us that not all weight spaces of $M^{\prime}\left(\dot{c}_{2}\right)$ are finite dimensional.

THEOREM 3.2. Let $\dot{c}_{2} \neq 0$. Then the vectors $L_{(-1,-1)} L_{(1,-1)} v, L_{(-2,-1)} L_{(2,-1)} v, \ldots$, $L_{(-n,-1)} L_{(n,-1)} v \in M_{(0,-2)}^{\prime}$ of $M^{\prime}\left(\dot{c}_{2}\right)$ are linearly independent for all positive integer $n$.

PROOF. Suppose there was a linear relation

$$
\sum_{i=1}^{n} a_{i} L_{(-i,-1)} L_{(i,-1)} v=0,
$$

where $a_{i} \in \mathbb{K}$. For any $k>n$, applying $L_{(k .1)}$ to (3.4) and using (3.3) we deduce that $\sum_{i=1}^{n} a_{i}(k-i)^{2} L_{(k,-1)} v=0$, for all $k>n$, that is,

$$
\sum_{i=1}^{n} a_{i}(k-i)^{2}=0, \quad \forall k>n .
$$


Since the left-hand side of (3.5) is a polynomial in $k$, it holds for all $k \in \mathbb{Z}$. Applying $L_{(k, 1)}(1 \leq k \leq n)$ to (3.4) and using (3.5) for these $k$ we deduce that

$$
\sum_{i=1}^{n} a_{i}(k-i)^{2} L_{(k,-1)} v+a_{k} \dot{c}_{2} L_{(k,-1)} v=0,
$$

which gives $a_{k}=0$. This completes the proof.

From Theorems 3.1 and 3.2, we know that not all weight spaces of any $G$-graded irreducible $\bar{L}$-modules (except for trivial one) given from $M\left(\dot{c}_{1}, \dot{c}_{2}, \prec\right)$ are finite dimensional.

If the index group $G \simeq \mathbb{Z}^{n}$ with $n>2$, we can define Verma modules $M\left(\dot{c}_{1}, \ldots, \dot{c}_{n}, \prec\right)$ over Virasoro-like algebras $\bar{L}(G)$ with respect to a total order ' $\prec$. We do not study the irreducibility of these Verma modules here since in this case the order $\prec$ and the skew symmetric bilinear form $\phi$ will be very complicated and we do not think the corresponding statement as neat as Theorem 3.1. Certainly it is a very interesting problem.

\section{Acknowledgement}

The authors would like to thank the referee for the valuable comments and suggestions.

\section{References}

[1] Y. Billig and K. Zhao, 'Vertex operator representations of quantum tori at roots of unity', Comm. Contemp. Math. 6 (2004), 195-220.

[2] D. Z. Djokovic and K. Zhao, 'Some infinite-dimensional simple Lie algebras in characteristic 0 related to those of Block' J. Pure Appl. Algebra 127 (1998), 153-165.

[3] S. Eswara Rao and K. Zhao, 'Highest weight irreducible representations of rank 2 quantum tori', Math. Res. Lett. 11 (2004), 615-628.

[4] Y. Gao, 'Representations of extended affine Lie algebras coordinatized by certain quantum tori', Compositio Math. 123 (2000), 1-25.

[5] J. Hu, X. Wang and K. Zhao, 'Verma modules over generalized Virasoro algebras Vir[G]', J. Pure Appl. Algebra 177 (2003), 61-69.

[6] V. G. Kac, Infinite dimensional Lie algebras (Cambridge University Press, 1990).

[7] V. G. Kac and K. A. Raina, Bombay lectures on highest weight representations of infinite dimensional Lie algebras (World Sci., Singapore, 1987).

[8] E. Kirkman, C. Procesi and L. A. Small, 'q-analog for the Virasoro algebra'. Comm. Alg. 22 (1994), 3755-3774.

[9] V. Mazorchuk. 'Verma modules over generalized Witt algebras', Compositio Math. 115 (1999), $2 \mathrm{l}-35$. 
[10] R. V. Moody and A. Pianzola, Lie algebras with triangular decomposition (Wiley, New York, 1995).

[11] M. I. Zaytseva, 'The set of orderings on abelian groups', Uspekhi Mat. Nauk 7 (1953), 135-137.

[12] H. Zhang and K. Zhao, 'Representations of the Virasoro-like algebra and its $q$-analog', Comm. Alg. 24 (1996), 4361-4372.

[13] K. Zhao, 'Highest weight irreducible representations of the Virasora-like algebra', preprint (2002).

[14] —. 'Weyl type algebras from quantum tori', to appear in Comm. Contemp. Math.

Department of Mathematics

Qingdao University

Qingdao 266071

P. R. China

e-mail: wanxd@public.qd.sd.cn
Department of Mathematics Wilfrid Laurier University

Waterloo

Ontario N2L 3C5

Canada

and

Institute of Mathematics

Academy of Mathematics and System Sciences

Chinese Academy of Sciences

Beijing 100080

P. R. China

e-mail:kzhao@wlu.ca 
